\title{
ON DAMS OF FINITE CAPACITY
}

\author{
LAJOS TAKÁCS 1 \\ (Received 10 May 1966, revised 27 February 1967)
}

\section{Introduction}

We shall consider the following mathematical model of dams of finite capacity. In the time interval $(0, \infty)$ water is flowing into a dam (reservoir). Denote by $\chi(u)$ the total quantity of water flowing into the dam in the time interval $(0, u)$. The capacity of the dam is a finite positive number $h$. If the dam becomes full, the excess water overflows. Denote by $\delta(u)$ the total quantity of water demanded in the time interval $(0, u)$. If there is enough water in the reservoir the demand is satisfied, if there is not enough water the difference is supplied from elsewhere. Denote by $\eta(t)$ the content of the dam at time $t . \eta(0)$ is the initial content.

In many applications it is assumed that $\delta(u)=u, 0 \leqq u<\infty$. In this case there is a continuous release at a constant unit rate when the dam is not empty.

If either the input $\{\chi(u), 0 \leqq u<\infty\}$, or the demand, $\{\delta(u), 0 \leqq u<\infty\}$, or both are stochastic processes and if we consider their separable versions, then $\eta(t)$, the content of the dam at time $t$, is a random variable for all $t \geqq 0$. The random variable $\eta(t)$ is completely determined by $\eta(0)$, by $\xi(u)=\chi(u)-\delta(u)$ for $0 \leqq u \leqq t$ and by $h$. We always suppose that $\eta(0)$ and $\{\xi(u), 0 \leqq u<\infty\}$ are independent.

We note that $\{\xi(u), 0 \leqq u<\infty\}$ can also be interpreted as the process describing the fluctuations of the level of the dam provided that the level can vary in the interval $(-\infty, \infty)$, and $\{\eta(t), 0 \leqq t<\infty\}$ describes the fluctuations of the level of the dam if the level can vary only in the interval $[0, h]$, that is, the excess water overflows and if necessary auxiliary water is used to ensure that the level never decreases below 0 . This latter interpretation permits us also to consider more general types of processes $\{\xi(u), 0 \leqq u<\infty\}$ than we mentioned above.

In this paper we shall find the limiting distribution of $\eta(t)$ as $t \rightarrow \infty$ when $\{\xi(u), 0<u<\infty\}$ is a real-valued, separable stochastic process with stationary independent increments for which the sample functions have no

1 This research was sponsored by the National Science Foundation under Contract No. NSF-GP-4866 at Columbia University. 
negative jumps and vanish at $u=0$ with probability 1 . In this case $\{\eta(t), 0 \leqq t<\infty\}$ is a Markov process. If we exclude the trivial case $\boldsymbol{P}\{\xi(u)=0\}=1$ for $u \geqq 0$, then the limiting distribution $\lim _{t \rightarrow \infty} \boldsymbol{P}\{\eta(t) \leqq x\}$ exists and is independent of the distribution of $\eta(0)$. We note that if $P\{\xi(u) \geqq 0\}=1$ for all $u \geqq 0$, then $\lim _{t \rightarrow \infty} \boldsymbol{P}\{\eta(t)=h\}=1$ and if $\boldsymbol{P}\{\xi(u) \leqq 0\}=1$ for all $u \geqq 0$, then $\lim _{t \rightarrow \infty} \boldsymbol{P}\{\eta(t)=0\}=1$ provided that $P\{\xi(u)=0\}<1$ for all $u>0$.

\section{The limiting distribution of the content of the dam}

If we suppose that $\{\xi(u), 0 \leqq u<\infty\}$ is a real-valued stochastic process with stationary independent increments for which the sample functions have no negative jumps and vanish at $u=0$ with probability 1 , then

$$
\boldsymbol{E}\left\{e^{-s \xi(u)}\right\}=e^{u \Phi(s)}
$$

exists for $\operatorname{Re}(s) \geqq 0$ and the most general form of $\Phi(s)$ is given by

$$
\Phi(s)=a s+\frac{1}{2} \sigma^{2} s^{2}-\int_{0}^{\infty}\left(1-e^{-s x}-\frac{s x}{1+x^{2}}\right) d N(x)
$$

where $a$ is a real constant, $\sigma^{2}$ is a nonnegative constant, $N(x), 0<x<\infty$, is a nondecreasing function of $x$ satisfying the requirements $\lim _{x \rightarrow \infty} N(x)=0$ and

$$
\int_{0}^{1} x^{2} d N(x)<\infty
$$

We note that if

$$
\int_{0}^{1} x d N(x)<\infty,
$$

then (2) can be reduced to the following form

$$
\Phi(s)=a s+\frac{1}{2} \sigma^{2} s^{2}-\int_{0}^{\infty}\left(1-e^{-8 x}\right) d N(x)
$$

where, in general, the constant $a$ is not the same as in (2).

We shall exclude the trivial cases mentioned in the Introduction and suppose that $\boldsymbol{P}\{\xi(u)>0\}>0$ and $\boldsymbol{P}\{\boldsymbol{\xi}(u)<0\}>0$ for all $u>0$. If a process $\{\xi(u), 0 \leqq u<\infty\}$ has these properties, then we say that it belongs to the class $D$. If $\Phi(s)$ is given by (2) and $\int_{0}^{1} x d N(x)=\infty$, then $\{\xi(u), 0 \leqq u<\infty\}$ belongs to $D$. If $\Phi(s)$ is given by $(5)$, when necessarily $\int_{0}^{1} x d N(x)<\infty$, then $\{\xi(u), 0 \leqq u<\infty\}$ belongs to $D$ if and only if either $\sigma^{2}>0$ or $a>0$ and $N(x) \not \equiv 0$. (Cf. G. Baxter and J. M. Shapiro [1].)

THEOREM 1. Let $\{\xi(u), 0 \leqq u \leqq \infty\}$ be a real-valued, separable stochastic process with stationary independent increments for which the sample functions 
have no negative jumps and vanish at $u=0$ with probability 1 . Suppose that $\{\xi(u), 0 \leqq u<\infty\}$ belongs to the class $D$. Let

$$
\boldsymbol{E}\left\{e^{-s \xi(u)}\right\}=e^{u \Phi(s)}
$$

for $\operatorname{Re}(s) \geqq 0$. Then the limiting distribution

$$
\lim _{t \rightarrow \infty} \boldsymbol{P}\{\eta(t) \leqq x\}=W(x) / W(h) \quad(0 \leqq x \leqq h)
$$

exists, is independent of the distribution of $\eta(0)$ and

$$
\int_{0}^{\infty} e^{-s x} W(x) d x=1 / \Phi(s)
$$

for $\operatorname{Re}(s)>\omega$ where $\omega$ is the largest nonnegative real root of $\Phi(s)=0$.

Before proving this theorem we shall give some examples for its applications.

\section{Examples}

1. Suppose that $\xi(u)=\chi(u)-u$ for $0 \leqq u<\infty$, where $\{\chi(u), 0 \leqq u<\infty\}$ is a separable stochastic process with stationary independent increments for which almost all sample functions are nondecreasing step functions vanishing at $u=0$. The trivial case $\boldsymbol{P}\{\chi(u)=0\}=1$ for $u \geqq 0$ is excluded. For such processes

$$
\Phi(s)=s-\int_{0}^{\infty}\left(1-e^{-s x}\right) d N(x)
$$

where

$$
\int_{0}^{1} x d N(x)<\infty .
$$

In this case $E\{\chi(u)\}=\rho u$ where

$$
\rho=\int_{0}^{\infty} x d N(x)
$$

is a positive number (possibly $\infty$ ).

Now

$$
\int_{0}^{\infty} e^{-8 x} W(x) d x=1 / \Phi(s)
$$

for $\operatorname{Re}(s)>\omega$. If $\rho \leqq 1$, then $\omega=0$, whereas if $\rho>1$, then $\omega>0$.

If $\rho \neq 1$, then by inversion we obtain that

$$
W(x)=\frac{e^{\omega x}}{\Phi^{\prime}(\omega)}-\int_{+0}^{\infty} d_{u} \boldsymbol{P}\{\chi(u) \leqq u+x\}
$$

where $d_{u} \boldsymbol{P}\{\chi(u) \leqq u+x\}=\boldsymbol{P}\{u+x \leqq \chi(u) \leqq u+x+d u\}$.

If $\boldsymbol{P}\{\chi(u) \leqq x\}$ has a density function, then (13) reduces to 


$$
W(x)=\frac{e^{\omega x}}{\Phi^{\prime}(\omega)}-\int_{0}^{\infty} \frac{\partial \boldsymbol{P}\{\chi(u) \leqq u+x\}}{\partial x} d u .
$$

If $\chi(u)$ is a discrete random variable, then (13) reduces to

$$
W(x)=\frac{e^{\omega x}}{\Phi^{\prime}(\omega)}-\sum_{\mathbf{0}<u<\infty} \boldsymbol{P}\{\chi(u)=u+x\} .
$$

If $x<0$, then the right hand side of (13) is 0 .

If $\rho$ is a finite positive number, then we can also obtain $W(x)$ in the following way. If $0<\rho<\infty$, then

$$
H^{*}(x)=\frac{\int_{0}^{x} N(y) d y}{\int_{0}^{\infty} N(y) d y} \quad(0 \leqq x<\infty)
$$

is a distribution function of a nonnegative random variable and for $\operatorname{Re}(s) \geqq 0$

$$
\Phi(s)=s\left[\mathbf{1}-\rho \psi^{*}(s)\right]
$$

where

$$
\psi^{*}(s)=\int_{0}^{\infty} e^{-s x} d H^{*}(x)
$$

Since

$$
\frac{1}{\Phi(s)}=\frac{1}{s\left[1-\rho \psi^{*}(s)\right]}=\frac{1}{s} \sum_{n=0}^{\infty} \rho^{n}\left[\psi^{*}(s)\right]^{n}
$$

for $\operatorname{Re}(s)>\omega$, by inversion we get that

$$
W(x)=\sum_{n=0}^{\infty} \rho^{n} H_{n}^{*}(x),
$$

where $H_{n}^{*}(x)$ is the $n$-th iterated convolution of $H^{*}(x)$ with itself; $H_{0}^{*}(x)=1$ if $x \geqq 0$ and $H_{0}^{*}(x)=0$ if $x<0$.

Accordingly, if we know $\boldsymbol{P}\{\chi(u) \leqq x\}$ for $0 \leqq u<\infty$ or $H^{*}(x)$, then by (13) or by (20) we can find $W(x)$. Now we shall consider some important particular cases.

(i) Compound Poisson input. In (9) let

$$
N(x)=-\lambda[1-H(x)], \quad(0<x<\infty)
$$

where $\lambda$ is a positive constant and $H(x)$ is the distribution function of a nonnegative random variable. Then

$$
\Phi(s)=s-\lambda[1-\psi(s)],
$$

where $\psi(s)$ is the Laplace-Stieltjes transform of $H(x)$. In this case 


$$
\boldsymbol{P}\{\chi(t) \leqq x\}=\sum_{n=0}^{\infty} e^{-\lambda t} \frac{(\lambda t)^{n}}{n !} H_{n}(x),
$$

where $H_{n}(x)$ denotes the $n$-th iterated convolution of $H(x)$ with itself; $H_{0}(x)=1$ if $x \geqq 0$ and $H_{0}(x)=0$ if $x<0$. Now $\rho=\lambda \alpha$, where $\alpha$ is the mean of the distribution function $H(x)$. If $\rho$ is a finite positive number, then by (16)

$$
H^{*}(x)=\frac{1}{\alpha} \int_{0}^{x}[1-H(y)] d y
$$

for $0 \leqq x<\infty$. By using (23) or (24) we can obtain $W(x)$ by (13) or (20) respectively.

This process has been considered before by J. Gani and N. U. Prabhu [2] in the case when $H(x)=1$ for $x \geqq \alpha$ and $H(x)=0$ if $x<\alpha$ and by B. Weesakul and G. Yeo [5] in the case when $H(x)=1-e^{-x / \alpha}$ for $x \geqq 0$.

(ii) Stable input. In (9) let

$$
N(x)=-\frac{1}{\Gamma(1-c) x^{c}}, \quad(0<x<\infty)
$$

where $0<c<1$. Then

$$
\Phi(s)=s-s^{c} .
$$

In this case

$$
\boldsymbol{P}\{\chi(t) \leqq x\}=\int_{0}^{x t^{-1 / c}} f_{c}(y) d y
$$

for $x \geqq 0$, where

$$
f_{c}(x)=\sum_{n=1}^{\infty} \frac{(-1)^{n-1} \Gamma(n c+1) \sin n c \pi}{n ! x^{n c+1}}
$$

In particular,

$$
f_{\frac{1}{2}}(x)=\frac{1}{\sqrt{4 \pi x^{3}}} e^{-1 / 4 x} .
$$

Now $\rho=\infty$ and $\omega=1$. Thus by (13)

$$
W(x)=\frac{e^{x}}{1-c}-\int_{0}^{\infty} f_{c}\left(\frac{x+u}{u^{1 / c}}\right) \frac{d u}{u^{1 / c}}
$$

for $x \geqq 0$. We have also

$$
W(x)=\sum_{n=0}^{\infty} \frac{x^{n(1-c)}}{\Gamma(n(1-c)+1)}
$$

for $x \geqq 0$.

(iii) Generalized stable input. In (9) let

$$
N(x)=-\frac{c}{\Gamma(1-c)} \int_{x}^{\infty} e^{-\mu y} \frac{d y}{y^{c+1}}, \quad(0<x<\infty)
$$


where $0<c<1$ and $\mu \geqq 0$. If $\mu=0$ in (32), we get (25). Then

$$
\Phi(s)=s+\mu^{c}-(s+\mu)^{c}
$$

and

$$
\boldsymbol{P}\{\chi(t) \leqq x\}=e^{t \mu^{c}} \int_{0}^{x t^{-1 / c}} e^{-\mu t^{1 / c} y} f_{\mathrm{c}}(y) d y
$$

for $x \geqq 0$, where $f_{c}(x)$ is defined by (28). Now $\rho=c \mu^{c-1}$. If $\rho \neq 1$, then $W(x)$ is given by (13). If $\rho$ is a finite positive number, then

$$
\frac{d H^{*}(x)}{d x}=\frac{\mu^{1-c}}{\Gamma(1-c)} \int_{x}^{\infty} e^{-\mu y} \frac{d y}{y^{c+1}}
$$

and $W(x)$ can also be obtained by (20).

(iv) Gamma input. In (9) let

$$
N(x)=-\int_{x}^{\infty} e^{-\mu \nu} \frac{d y}{y}, \quad(0<x<\infty)
$$

where $\mu$ is a positive constant. Then

$$
\Phi(s)=s-\log \left(1+\frac{s}{\mu}\right)
$$

and

$$
\boldsymbol{P}\{\chi(t) \leqq x\}=\frac{1}{\Gamma(t)} \int_{0}^{\mu x} e^{-v^{t-1}} d y
$$

for $x \geqq 0$. Now $\rho=1 / \mu$ and

$$
\frac{d H^{*}(x)}{d x}=\mu \int_{\mu x}^{\infty} e^{-y} \frac{d y}{y}
$$

for $x>0$. Both (13) and (20) can be used to obtain $W(x)$.

This process has been introduced by P. A. P. Moran [2], but he did not give the limiting distribution for finite dams.

2. Suppose that $\{\xi(u), 0 \leqq u<\infty\}$ has stationary independent increments and $\Phi(s)$ is given by (2) with $\sigma^{2}=0$ and $N(x)=-1 / x$ for $0<x<\infty$. Then

$$
\Phi(s)=a s-\int_{0}^{\infty}\left(1-e^{-s x}-\frac{s x}{1+x^{2}}\right) \frac{d x}{x^{2}}=s[a-1+\gamma+\log s],
$$

where $\gamma=0.5772157 \cdots$ is the Euler constant. Now $\omega=e^{1-a-\gamma}$ and

$$
W(x)=\frac{1}{\omega} \int_{0}^{\infty} \frac{(\omega x)^{u+1}}{\Gamma(u+2)} d u
$$

for $x \geqq 0$. 
3. Suppose that $\{\xi(u), 0 \leqq u<\infty\}$ is a Brownian motion process with $E\{\xi(u)\}=\alpha u$ and $\operatorname{Var}\{\xi(u)\}=\sigma^{2} u$. Then

$$
\boldsymbol{P}\left\{\frac{\xi(u)-\alpha u}{\sqrt{\sigma^{2} u}} \leqq x\right\}=\frac{1}{\sqrt{2 \pi}} \int_{-\infty}^{x} e^{-y^{2} / 2} d y
$$

and

$$
\Phi(s)=-\alpha s+\frac{1}{2} \sigma^{2} s^{2} .
$$

Now $\omega=0$ if $\alpha \leqq 0$ and $\omega=2 \alpha / \sigma^{2}$ if $\alpha>0$. Since

$$
\int_{0}^{\infty} e^{-\alpha x} W(x) d x=\frac{2}{s\left(\sigma^{2} s-2 \alpha\right)}=\frac{1}{\alpha}\left[\frac{\sigma^{2}}{\sigma^{2} s-2 \alpha}-\frac{1}{s}\right]
$$

for $\operatorname{Re}(s)>\omega$, we get by inversion that for $x \geqq 0$

$$
W(x)=\frac{1}{\alpha}\left(e^{2 \alpha x / \sigma^{2}}-1\right) \quad \text { if } \alpha \neq 0,
$$

and

$$
W(x)=2 x / \sigma^{2} \quad \text { if } \alpha=0 .
$$

\section{A discrete storage process}

First we shall prove an auxiliary theorem.

Lemma. Let $\pi_{j}, j=0,12, \cdots$, be a probability distribution with expectation

$$
\gamma=\sum_{j=0}^{\infty} j \pi_{j}
$$

$(0 \leqq \gamma \leqq \infty)$ and generating function

$$
\pi(z)=\sum_{j=0}^{\infty} \pi_{j} z^{j}
$$

for $|z| \leqq 1$. If $\pi_{0}>0$, then the solution of the following system of linear equations

$$
Q_{k}=\sum_{j=0}^{k} \pi_{j} Q_{k-j+1}, \quad k=0,1,2, \cdots,
$$

is given by the generating function

$$
\sum_{k=1}^{\infty} Q_{k} z^{k}=\frac{Q_{0} z}{\pi(z)-z}
$$

which is convergent if $|z|<\delta$ where $z=\delta$ is the smallest nonnegative real root of $\pi(z)=z$. If $\gamma \leqq 1$, then $\delta=1$, whereas if $\gamma>1$, then $0<\delta<1$. 
Proof. If $\gamma \leqq 1$ and $\pi_{0}>0$, then $\pi(z)=z$ has exactly one root $z=1$ in the unit circle $|z| \leqq 1 \quad(z=1$ is a simple root if $\gamma<1$ and $z=1$ is a double root if $\gamma=1$ ). If $\gamma>1$, then $\pi(z)=z$ has two roots $z=1$ and $z=\delta$, where $0<\delta<1$, in the unit circle $|z| \leqq 1$. This can be proved directly or by using Rouchés theorem. If we form the generating function of (49), then we get (50) and (50) is convergent if $|z|<\delta$.

Now we define a storage process in the following way. Let $\{v(u), 0 \leqq u<\infty\}$ be a Poisson process of density $\lambda$ and let $v_{1}, v_{2}, \cdots, v_{n}, \cdots$ be a sequence of mutually independent and identically distributed random variables with distribution $\boldsymbol{P}\left\{\boldsymbol{v}_{n}=j\right\}=\pi_{j}, j=0,1,2, \cdots$, and let $\left\{\boldsymbol{v}_{n}\right\}$ be independent of $\{v(u), 0 \leqq u<\infty\}$. Set $N_{n}=v_{1}+v_{2}+\cdots+v_{n}$ for $n=1,2, \cdots$ and $N_{0}=0$. Define $\xi(u)=N_{\nu(u)}-v(u)$ for $0 \leqq u<\infty$. Then $\{\xi(u), 0 \leqq u<\infty\}$ is a compound Poisson process. Now consider a store which may contain $0,1,2, \cdots, m$ items. Let $\eta(0)$ be the initial stock size. Suppose that starting from its initial value the stock size varies according to the process $\{\xi(u), 0 \leqq u<\infty\}$; however, the stock size cannot increase over $m$ or decrease below 0 . That is, an overflow may occur if the store is full and demands are not satisfied if the store is empty. This is the discrete version of the dam process defined in the Introduction. Denote by $\eta(t)$ the stock size at time $t$, and suppose that $\eta(0)$ and $\{\xi(u), 0 \leqq u<\infty\}$ are independent.

THEOREM 2. If $\pi_{0}>0$, then

$$
\lim _{t \rightarrow \infty} \boldsymbol{P}\{\eta(t) \leqq k\}=Q_{k} / Q_{m}
$$

for $k=0,1, \cdots, m$, where $Q_{k}, k=0,1,2, \cdots$, is defined in the Lemma with an arbitrary $Q_{0} \neq 0$. The limit (51) is independent of the distribution of $\eta(0)$.

Proof. Now $\{\eta(t), 0 \leqq t<\infty\}$ is a Markov process with state space $I=\{0,1, \cdots, m\}$. If we suppose that $\pi_{0}>0$, then it can easily be seen that $\lim _{t \rightarrow \infty} \boldsymbol{P}\{\eta(t) \leqq k\}=Q_{k}^{*}$ exists and is independent of the distribution of $\eta(0)$. For $t \geqq 0$ and $k=0,1, \cdots, m-1$, we have

$$
\boldsymbol{P}\{\eta(t+u) \leqq k\}=(1-\lambda u) \boldsymbol{P}\{\eta(t) \leqq k\}+\lambda u \sum_{j=0}^{k} \pi_{j} \boldsymbol{P}\{\eta(t) \leqq k-j+1\}+o(u)
$$

and $\boldsymbol{P}\{\eta(t) \leqq m\}=1$. If we form the limit $t \rightarrow \infty$ in (52), then it follows that

$$
Q_{k}^{*}=\sum_{j=0}^{k} \pi_{j} Q_{k-j+1}^{*}
$$

for $k=0,1, \cdots, m-1$ and $Q_{m}^{*}=1$. If we compare (49) and (53), then we can conclude immediately that $Q_{k}^{*}=Q_{k} / Q_{m}$ for $k=0,1, \cdots, m$. This completes the proof of Theorem 2 . 


\section{Proof of Theorem 1}

Consider the process $\{\xi(u), 0 \leqq u<\infty\}$ introduced in Section 4 . However, we suppose that the probabilities $\pi_{j}(j=0,1,2, \cdots)$ also depend on $\lambda$. We use the notation $\pi_{j}^{(\lambda)}, \pi^{(\lambda)}(z)$ and $Q_{k}^{(\lambda)}$ indicating this dependence. Also we use the notation $\left\{\xi_{\lambda}(u), 0 \leqq u<\infty\right\}$ for the corresponding process. It is easy to see that we can choose the distributions $\left\{\pi_{j}^{(\lambda)}, j=0,1,2, \cdots\right\}$ and a normalizing factor, say $\alpha / \sqrt{ } \lambda$, such that, as $\lambda \rightarrow \infty$, the finite dimensional distributions of the process $\left\{\alpha \xi_{\lambda}(u) / \sqrt{ } \lambda, 0 \leqq u<\infty\right\}$ converge to the corresponding finite dimensional distributions of the process $\{\xi(u)$, $0 \leqq u<\infty\}$ defined in Theorem 1 . For if we denote by $\pi^{(\lambda)}(z)$ the generating function of $\left\{\pi_{j}^{(\lambda)}, j=0,1,2, \cdots\right\}$, then for $\operatorname{Re}(s) \geqq 0$

$$
\boldsymbol{E}\left\{e^{-s \xi_{\lambda}(u)}\right\}=e^{u \Phi_{\lambda}(s)},
$$

where

$$
\Phi_{\lambda}(s)=\lambda\left[e^{s} \pi^{(\lambda)}\left(e^{-s}\right)-1\right],
$$

and we can choose the distributions $\left\{\pi_{j}^{(\lambda)}\right\}$ such that

$$
\lim _{\lambda \rightarrow \infty} \Phi_{\lambda}\left(\frac{\alpha s}{\sqrt{ } \lambda}\right)=\Phi(s)
$$

for $\operatorname{Re}(s) \geqq 0$, where $\Phi(s)$ is defined by (2). Hence the statement follows.

If we denote by $\left\{\eta_{\lambda}(u), 0 \leqq u<\infty\right\}$ the storage process corresponding to $\left\{\xi_{\lambda}(u), 0 \leqq u<\infty\right\}$ and suppose that

$$
m=[\sqrt{ } \lambda h / \alpha] \text { and } \eta_{\lambda}(0)=[\sqrt{ } \lambda \eta(0) / \alpha],
$$

then we can conclude that as $\lambda \rightarrow \infty$ the finite dimensional distributions of the process $\left\{\alpha \eta_{\lambda}(u) / \sqrt{ } \lambda, 0 \leqq u<\infty\right\}$ converge to the corresponding finite dimensional distributions of the process $\{\eta(u), 0 \leqq u<\infty\}$ considered in Theorem 1.

Accordingly we can conclude that for $0 \leqq x \leqq h$

$$
\lim _{t \rightarrow \infty} \boldsymbol{P}\{\eta(t) \leqq x\}=\lim _{\lambda \rightarrow \infty} \frac{Q_{k}^{(\lambda)}}{Q_{m}^{(\lambda)}}
$$

whenever $k=[\sqrt{ } \lambda x / \alpha]$ and $m=[\sqrt{ } \lambda h / \alpha]$. Thus,

$$
\lim _{t \rightarrow \infty} \boldsymbol{P}\{\eta(t) \leqq x\}=W(x) / W(h)
$$

for $0 \leqq x \leqq h$, where

$$
\int_{0}^{\infty} e^{-s x} W(x) d x=\lim _{\lambda \rightarrow \infty} \frac{\alpha}{\sqrt{ } \lambda} \sum_{k=1}^{\infty} Q_{k}^{(\lambda)} e^{-s k \alpha / \sqrt{ } \lambda}=\lim _{\lambda \rightarrow \infty} \frac{\alpha}{\sqrt{ } \lambda} \frac{Q_{0}^{(\lambda)}}{\Phi_{\lambda}\left(\frac{\alpha s}{\sqrt{ } \lambda}\right)}
$$


If we choose $Q_{0}^{(\lambda)}=\sqrt{ } \lambda / \alpha$, then we get

$$
\int_{0}^{\infty} e^{-s x} W(x) d x=1 / \Phi(s)
$$

for $\operatorname{Re}(s)>\omega$, where $\Phi(s)$ is defined by $(2)$. This completes the proof of Theorem 1.

Note. Finally, we would like to note that the limiting distribution of the content of the dam can also be expressed in the following way

$$
\lim _{t \rightarrow \infty} \boldsymbol{P}\{\eta(t) \leqq x\}=\boldsymbol{P}\{\xi(u) \leqq x \text { for } 0 \leqq u \leqq \theta(h-x)\}
$$

for $0 \leqq x \leqq h$, where

$$
\theta(x)=\inf \{u: \xi(u) \leqq-x \text { and } 0 \leqq u<\infty\}
$$

and $\theta(x)=\infty$ if $\xi(u)>-x$ for all $u \geqq 0$. (See reference [4].)

\section{References}

[1] G. Baxter and J. M. Shapiro, 'On bounded infinitely divisible random variables', Sankhyā, 22 (1960), 253-260.

[2] J. Gani and N. U. Prabhu, 'Continuous time treatment of a storage problem', Nature, 182 (1958), 39-40.

[3] P. A. P. Moran, 'A probability theory of a dam with continuous release', Quart. Journ. Math. Oxford, 7 (1956), 130-137.

[4] L. Takács, Combinatorial Methods in the Theory of Stochastic Processes (John Wiley and Sons, New York, 1967).

[5] B. Weesakul and G. Yeo, 'Some problems in finite dams with an application to insurance risk', Zeitschrift für Wahrscheinlichkeitstheorie 2 (1963), 135-146.

Department of Mathematics

Case Western Reserve University

Cleveland, Ohio, U.S.A. 